\title{
Sensing Users' Emotional Intelligence in Social Networks
}

\author{
Xiangyu Wei, Guangquan Xu, Hao Wang, Yongzhong He, Zhen Han, Wei Wang
}

\begin{abstract}
Social networks have integrated into daily lives of most people in the way of interactions and of lifestyles. The users' identity, relationships, or other characteristics can be explored from the social networking data, in order to provide personalized services to the users. In this work, we focus on predicting the user's emotional intelligence (EI) based on the social networking data. As an essential facet of users' psychological characteristics, EI plays an important role on well-being, interpersonal relationships, and overall success in people's life. Perception of emotional intelligence contributes to predicting one's behavior or group behavior. Most existing work on predicting people's emotional intelligence is based on questionnaires that may collect dishonest answers or unconscientious responses, thus leading in potentially inaccurate prediction results. In this work, we are motivated to propose emotional intelligence prediction models based on the sentiment analysis of social networking data. The models are represented by four dimensions including self-awareness, self-regulation, self-motivation and social relationships. The EI of a user is then measured by four numerical values or the sum of them. In the experiments, we predict the EIs of over a hundred thousand users based on one of the largest social networks of China, Weibo. The predicting results demonstrate the effectiveness of our models. The results show that the distribution of the four EI's dimensions of users is roughly normal. The results also indicate that EI scores of females are generally higher than males'. This is consistent with previous findings. In addition, the four dimensions of EI are correlated. We finally analyze the advantages and the disadvantages of our models in predicting users' EI with social networking data.
\end{abstract}

Index Terms-Emotional intelligence, Sentiment analysis, User profile, Social networks.

\section{INTRODUCTION}

$\mathbf{S}$ OCIAL networks such as Twitter, Weibo, Instagram are used everyday to express personal thoughts, which enable researchers to gain valuable insight into a large number of personal opinions. The views on persons and events can

Xiangyu Wei, Yongzhong He, Zhen Han, Wei Wang are with Beijing Key Laboratory of Security and Privacy in Intelligent Transportation, Beijing Jiaotong University, 100044 Beijing, China (e-mail: \{weixiangyu, yzhhe, zhan, wangwei1\}@bjtu.edu.cn). Wei Wang is also with the Division of Computer, Electrical and Mathematical Sciences \& Engineering (CEMSE), King Abdullah University of Science and Technology (KAUST), Thuwal 23955-6900, Saudi Arabia.

Guangquan Xu is with Tianjin Key Laboratory of Advanced Networking, College of Intelligence and Computing, Tianjin University, 300350 Tianjin, China (e-mail:losin@tju.edu.cn).

Hao wang is with the Department of Computer Science, Norwegian University of Science and Technology, 7491 Trondheim, Norway (e-mail: hawa@ntnu.no).

The work reported in this paper was supported in part by National Key R\&D Program of China, under Grant 2017YFB0802805, and in part by the Natural Science Foundation of China, under Grant U1736114.

Corresponding author: Wei Wang and Hao Wang

Manuscript received June 30, 2019 reflect the user's emotional intelligence. Understanding EI is in favor of perceiving individual behavior changes and predicting group behavior. However, the emerging social networking platforms as well as the massive informative data users input have aroused concerns for users' privacy, as it is not difficult to analyze users' characteristics and even disclose users' private information based on the social networking data.

Previous work [1]-[3] on users' attributions revolved around users' demographics, such as age, gender and location. In addition, many researchers [4]-[9] mined users' psychological traits through social networks. In spite of some work on employing artificial intelligence technology to conduct privacy analysis [10], [11] and abnormal behavior detection [12][21] or privacy protection and system security [22]-[26], we focus on the privacy disclosure based on the text published by users in social networks to provide an extra source of information. Moreover, there is no related work on predicting users' emotional intelligence based on social networks.

Thorndike et al. [27] proposed the concept of Social Intelligence in 1920 and Alexander et al. [28] proposed the concept of Non-intellectual Factors in 1938. In 1987, Gardner et al. [29] proposed Multiple Intelligence. In fact, the concept of emotional intelligence develops from these early ideas and theories. In 1990, American psychologists Salovey and Mayer [30] reinterpreted emotional intelligence and formally proposed systematic theory. Bar-On also proposed his own definition. Bar-On [31] first proposed the concept of the emotional quotient in 1985 and published his Emotional Quotient Inventory. According to the Bar-On model, each of these metafactorial components of EI comprises a number of closely related abilities, skills and behaviors.

Emotional intelligence [30] is a type of user's characteristics that involve the ability to monitor one's own emotions as well as others' so as to discriminate them and to use the information to guide one's thinking and actions. Emotional intelligence [32] includes four dimensions: emotional perception and expression, emotional thinking, emotional understanding, and emotional management. We consider the predictive models according to those definitions of emotional intelligence. In this paper, we hypothesize that we can continuously track emotions of the users who are inclined to publishing tweets or share live events. If users prefer to use the same or similar sentiment words, the ability of self-awareness is similar. Self-regulation is different between users who have different frequency of emotional fluctuations. Users who are in a positive emotional state have different ability of self-motivation from users who always have negative state. And emotional intelligence has an important impact on social relationships. 
There also exist related work on predicting validity of emotional intelligence in various scenarios [33]-[35], and many studies reveal that emotional intelligence has an important impact on motivation, emotional regulation, stress, decisionmaking, work performance and job satisfaction [31], [36], [37].

Previous approaches used to estimate people's emotional intelligence were based on questionnaires. There are some factors affecting the measurement in the self-reported questionnaire. For example, the respondent has a response set, such as extreme, avoidance and carelessness. Or rather, respondents fail to know their own situations and understand the grading standards. Meanwhile, respondents' answers to some questions were affected by unstable factors, such as emotions and motives at that time, which means that the test results may not truly reflect the psychological level of the person. Moreover, emotional intelligence is constantly changing as the living environment changes [38]. In contrast, social networks provide a good platform for the measurement of users' EIs, as it contains rich information for profiling a very big number of users and most users in social networks are honest to convey themselves.

In this work, we propose a prediction model of emotional intelligence based on the sentiment analysis of social networking data. Our work is extended based on our previous work in [39] where we briefly introduced the models of predicting users' emotional intelligence and the experimental results in terms of the distribution of the users' emotional intelligence and their gender differences. In this work, we additionally provide correlation coefficient analysis to compare the relationship of the four dimensions of EI. Furthermore, the algorithmic description has been enhanced. Our extended version also contains comprehensive discussion on classifying users from the perspective of emotional intelligence.

The models are represented by four dimensions including self-awareness, self-regulation, self-motivation and social relationships. The EI of a user is then measured by four numerical values or the sum of them. In the experiments, we predict the EIs of over a hundred thousand users based on one of the largest social networks of China, Weibo. The predicting results demonstrate the effectiveness of our models. In previous work, sentiment analysis is applied to agreement detection [40], and sarcasm identification [41], [42], stock prediction [43]. We implement the sentiment analysis to analyze users' text for predicting emotional intelligence.

We make the following contributions:

- We propose an emotional intelligence prediction models based on social networking data to measure users' ability to control emotions. The models includes self-awareness, self-regulation, self-motivation and social relationships according to the psycho-emotional intelligence theory. To the best of our knowledge, this is the first work towards predicting users' emotional intelligence based on social networking data.

- We test our models on one of the largest social networks in China, Weibo, and predict emotional intelligence of over a hundred thousand users of Weibo. The prediction results verify the effectiveness of our models. We also discuss and analyze the advantages and disadvantages of our models.

- We compare the topics among users with low scores and high scores and classify users from the perspective of emotional intelligence with the aim of further revealing the inherent law of users' emotional expression in social networks.

The reminder of this paper is organized as follows. The models for predicting emotional intelligence are proposed in Section II. The experiments and results are presented in Section III. We discuss and analyze the models and the results in Section IV. Finally, concluding remarks follow in Section $\mathrm{V}$.

\section{Prediction Models of Emotional Intelligence}

We propose an emotional intelligence prediction models which evaluate the user's emotional intelligence by analyzing the text and interactive information in social networks. Definitions of emotional intelligence include one or more of the key components, all of which are included in the BarOn model. We predict users' emotional intelligence based on the Weibo. Through the text that they published and the basic information including the number of comments, the number of retweet, publishing time, we carefully select a set of rules based on conceptualizations of emotional intelligence.

Thus, the models are based on four dimensions including self-awareness, self-regulation, self-motivation as well as social relationships according to the psycho-emotional intelligence theory. These four dimensions represent different aspects of emotional intelligence, including perceiving emotions, managing emotions, and interpersonal relationships.

The self-awareness(A) reflects the ability to accurately identify own emotions and appraisal and expression of emotions encompassing the ability to be sensitive to the slight variations between emotions. The categories and intensities of sentiment words in the text can reflect the user's sentiment. Through analyzing the emotional words of tweets published by users, we calculate the value of self-awareness.

The self-regulation $(\mathrm{R})$ includes the ability to manage disruptive emotions and impulses. Therefore, emotionally intelligent individuals often pay attention to harness emotions. The high mean of sentiment values, the low variance and users' emotional change are the measurement of the emotional instability or disorder of a user. We obtain the value of the self-regulation by the degree of sentiment changes.

The self-motivation(M) measures whether the user's emotions are always in a positive state and the recovery from disappointment is rapid. The self-motivation can be measured by the time of the recovery from the depressed stage to emotional stable stage and overall emotional state of the users.

The social relationships(S) measures the ability of infecting others' emotions, and the harmonization in the process of interpersonal interaction. Emotional intelligence helps individuals to behave appropriately, which allows them to maintain good relationships with others. The number of comments, the number of followers and the number of retweets can reflect the social relationships. We consider the number of the mood 
swings and average sentiment value as the factors that affect social relationships.

First, we need to set a time window for analyzing the text. The experimental time window is day. Assuming a time window contains $W$ sentences, the expect sentiment value in the time window $j$ is

$$
E_{j}=\frac{1}{W} \sum_{i=1}^{W} e_{i}
$$

where $e_{i}$ is the sentiment value of the sentence $i$ in the time window $j$.

Variance of sentiment values is defined as follows:

$$
\sigma_{j}=\frac{1}{W} \sum_{i=1}^{W}\left(e_{i}-E_{j}\right)^{2}
$$

Emotional change value is defined as follows:

$$
\delta_{j}=E_{j}-E_{j-1}
$$

Then we calculate the values of the four dimensions of emotional intelligence.

\section{A. Self-awareness}

The self-awareness is the ability to recognize own and others' emotions. There are 21 categories of sentiment words, and the each category has five intensities. The proportion of sentiment words in the whole text reflects the frequency of expressing emotions. The proportion of sentiment words with different categories reflects the richness of the user's emotions. The proportion of sentiment words with different intensities in the same category reflects the ability of identifying slight variations between emotions. In addition, whether the emotion can be expressed accurately or not is influenced by the user's current emotion. Therefore, the sentiment value of the text $E_{i}$ is also an important indicator of cognitive ability. The selfawareness is defined as follows:

$$
A=\frac{1}{k} \sum_{i=0}^{k}\left(\frac{w_{s}}{w_{n}}+\frac{w_{i}}{w_{c}}+\frac{w_{p}}{w_{s}}\right) E_{i}
$$

Where $w_{s}$ is the number of sentiment words, $w_{n}$ is the number of words. $w_{i}$ is the number of different intensities of sentiment words, $w_{c}$ is the number of the categories of the sentiment words. $E_{i}$ is the average sentiment value in the time windows $i . k$ is the number of time windows.

\section{B. Self-regulation}

The self-regulation reflects the ability to manage own emotions. The high value of self-regulation manifests the high mean of sentiment values and the low variance. When the variance of sentiment values of the user exceeds 0.1 in a time window, it indicates that the user's emotion has fluctuated. At the same time, the entropy is introduced to indicate the fluctuations of the user's emotions. The smaller the entropy is, the more stable the mood is and the less emotional fluctuations. Assuming $m$ represents the number of positive emotions of the user, and $n$ represents the number of negative emotions. The equation for entropy is

$$
\text { entropy }=\frac{-m}{m+n} \times \log \frac{m}{m+n}-\frac{n}{m+n} \times \log \frac{n}{m+n}
$$

The self-regulation is defined as follows:

$$
R=\frac{1}{k} \sum_{i=0}^{k} \frac{E_{i}+1}{\left(\sigma_{i} \times \text { entropy } \times \log t\right)+1}
$$

Where $t$ is the average time window intervals between the window where the variance of sentiment values does not exceed 0.1 and the average sentiment value is larger than 0.5 and the window where the mood swing appears. Tweets published by users reflect their sentiment and we consider that people's mood swings in a 28-day cycle. In other words, if the time of the users' mood swings is more than 28 days, we choose the 28 days as the time window intervals of the mood swings.

\section{Self-motivation}

The self-motivation can be measured by the time of the recovery from the depressed stage to emotional stable stage, and the proportion of positive emotions in the whole time. When the average sentiment value of a user in a window is less than 0.5 , the window can be viewed as a depressed stage. The $t_{l}$ is calculated by the average number of time window from less than 0.5 to more than 0.5 for the first time. The self-motivation is also affected by the user's current emotional level. Therefore, self-motivation is defined as follows:

$$
M=\left(\arctan \frac{1}{k} \sum_{i=0}^{k} \frac{w_{p} \times E_{i} \times 25}{w_{s} \times \log t_{l}}\right) \times 2 \div \pi
$$

Where $w_{p}$ is the number of positive words, $w_{s}$ is the number of the sentiment words. 25 is an amplification factor. We use $\pi$ to adjust values from 0 to 1 in the arctan function.

\section{Social Relationships}

The social relationships reflects the harmony of relationships and the ability of emotional contagion. We use the number of mood-swing, comments and retweet to measure how much one person influences another. In addition, when the emotional change value is greater than 0.2 , we view it as a mood swing. The number of comments and retweet are quantitative indicator to determine the influence of the text. Additionally, whether the text conveys a positive attitude to others can be considered. As the transmission of positive information, people can form a positive relationship. Therefore we add the average sentiment value to the formula. The social relationships is defined as follows:

$$
S=\left(\arctan \frac{1}{k} \sum_{i=1}^{k} \log \left(\frac{N_{c i} \times N_{t i}}{N_{d i}}\right) \times E_{i}\right) \times 2 \div \pi
$$

Where $N_{c i}$ is the ratio of the number of comments to the number of followers. $N_{t i}$ is a ratio that takes into the number of retweet and followers in the time window $i . N_{d i}$ represent the number of the mood swings. In order to achieve the overall 
convergence of the values, the values after the calculation are processed by the inverse tangent function. We standardize these scores from 0 to 1 .

\section{E. Measurement}

We calculate the values of the four dimensions of emotional intelligence through the Equation 4, the Equation 6, the Equation 7 and the Equation 8. The result is shown in Table I and the values of EI of a user after data standardization is shown in Figure 1.

There are two methods to measure users' EI. As shown in Table I, we use the sum of the four dimensions of EI as the first method. This method can reflect the user's comprehensive score, however the size of value of each dimension cannot be distinguished. The second method, shown in Figure 1, uses values of the four dimensions of EI to measure users' EI. This method can intuitively figure out the difference of each dimension.

\section{EXPERIMENTS AND RESULTS}

\section{A. Data set}

We measure users' EIs based on the weibo data that was collected by Fudan NLP Group ${ }^{1}$. The data set consists of 1.5 billion tweets and more than 2 million users. As shown in Table II, the user's information includes user identifier, number of comments, number of retweet, source, publishing time and post. In addition, we have the number of followers and the number of following. Weibo data is used as the training data set for naive Bayesian method.

We choose one hundred thousand users who have over 500 tweets and over 10 friends and followers, in order to measure their EI in the experiment. The number of micro blog is 105176341.

The micro-blog is very short (140 words), and there are @, tags, URLs and other noise information that are relatively unimportant for sentiment analysis in a single micro-blog. Thus, we carry out denoising and word segmentation.

\section{B. Chinese Sentiment Analysis}

In order to recognize the expression of emotions and calculate the value of self-awareness, we analyze the lexicon in the text published by the user through the Chinese emotional vocabulary [44]. The vocabulary containing 27466 sentiment words is divided into 7 major categories and 21 subcategories.

The major categories include happy, good, angry, sad, fearful, evil, shocked. 1, 3, 5, 7, and 9 denote different emotional intensities. 9 indicates the highest intensity and 1 is the lowest intensity.

Moreover, we expand the vocabulary by calculating the word semantic similarity through the words of HowNet and the word forest [45]. According to the distribution of the word, a dynamic weighting strategy of considering both HowNet and CiLin is used to calculate the word similarity.

For the sake of calculating the values of the four dimensions of EI, we should know each sentence's sentiment. Emoticons

\footnotetext{
${ }^{1}$ http://sma.fudan.edu.cn/index.html
}

in the user's post are closely related to the content. Accordingly, we extract emoticons to obtain the sentiment value of a sentence.

Firstly, the Sina weibo data is deduplicated and a total of 341,067 emoticons are extracted. We remove the error emoticons. Then, we choose emoticons with the number of occurrences more than 400 times. Finally, we obtain 1818 emoticons including some the traditional Chinese characters' emoticons. And after counting all the 1818 emoticons, we find that the appearance of the emoticons was consistent with the long tail distribution. As shown in Figure 2, the top $10 \%$ of emoticons account for $96.3 \%$ of all emoticons. Therefore, we manually annotate the top $10 \%$ of emoticons.

1 represents the positive emoticon and -1 represents the negative emoticon. Assuming that the number of the positive emoticon is $N_{p}$ and the number of the negative emoticon is $N_{n}$. The sentiment value of a sentence is as follows:

$$
s=\left\{\begin{array}{lll}
-1 & \text { for } & N_{p}<N_{n} \\
1 & \text { for } & N_{p} \geq N_{n}
\end{array}\right.
$$

As show in the Table III, after classification according to above the formula, the number of micro-blogs with positive sentiment is 14125 , and the number of micro-blogs with negative sentiment is 5793. Additionally, we have 7134 micro-blogs with positive sentiment and 8257 micro-blogs with negative sentiment that are manually annotated, including micro-blog emotional evaluation data set of China Computer Federation International Conference on Natural Language Processing and Chinese Computing (NLPCC) in $2014^{2}$. The data is imbalance. There are some methods about class-imbalance learning and classification algorithms comparison [46], [47]. We employ the down-sampling technique to make sure the classbalance. Those data sets are trained for sentiment analysis using the naive Bayesian method.

The naive Bayesian method is used to construct a text sentiment classifier. Based on the prior probability, we obtain the probability of observing different features under a given hypothesis. We define the category as

$$
C=\arg \max P\left(C_{j}\right) \prod_{i=1}^{n} P\left(w_{i}, C_{j}\right)^{w t\left(w_{i}\right)}
$$

Where $P\left(C_{j}\right)$ represents the prior probability of category $C_{j}, P\left(w_{i}, C_{j}\right)$ is the posterior probability of the characteristic word in the category $C_{j}, w t\left(w_{i}\right)$ is the weights of the word $w_{i}$ in the testing corpus.

The naive Bayesian method is used to classify the text sentiment in a positive probability. The positive probability which is greater than or equal 0.6 means a positive emotion. The positive probability which is less than 0.6 means a negative emotion.

\section{Experimental Result Analysis}

As described in Section II, we obtain the values of four dimensions of EI. In order to evaluate the validity of our models, we analyze the distribution of these values, the relationships between users' gender and EI, and the relationship of four dimensions of EI.

\footnotetext{
${ }^{2}$ http://tcci.ccf.org.cn/conference/2014/pages/page04_sam.html
} 


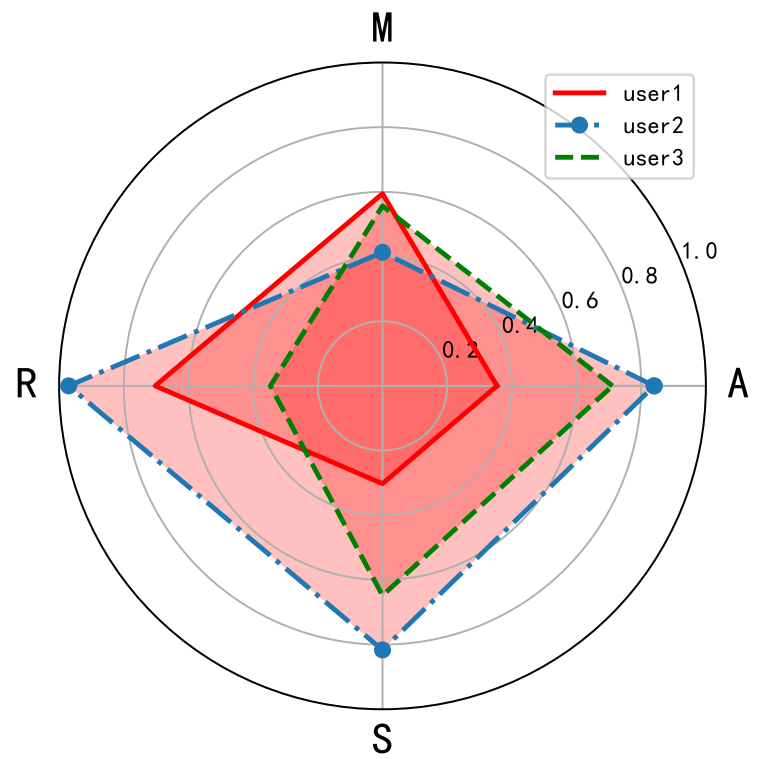

Fig. 1. The distribution of the values of EI. A represents self-awareness, M represents self-motivation, R represents self-regulation, and S represents social relationships. The values are the range for the four dimensions of EI of a user after data standardization. The shaded areas can be viewed as the sum of the four dimensions of emotional intelligence.

TABLE I

THE VALUES OF THE FOUR DIMENSIONS OF EMOTIONAL INTELLIGENCE.

\begin{tabular}{cccccc}
\hline \hline User & $\mathrm{A}$ & $\mathrm{M}$ & $\mathrm{R}$ & $\mathrm{S}$ & $\mathrm{sum}$ \\
\hline user1 & 0.65760 & 0.65899 & 1.26609 & 0.31275 & 2.8954 \\
user2 & 1.27210 & 0.52058 & 1.145383 & 0.809535 & 3.7361 \\
user3 & 1.10951 & 0.63020 & 1.01585 & 0.64672 & 3.404 \\
\hline
\end{tabular}

A represents self-awareness, $M$ represents self-motivation, $R$ represents self-regulation, and $\mathrm{S}$ represents social relationships.

TABLE II

WEIBO DATA

\begin{tabular}{cccccc}
\hline \hline userid & retweets & comments & source & time & text \\
\hline 1427652963 & 0 & 0 & iPhone & $\begin{array}{c}\text { 什么时候狠, 什么时候软也真是 } \\
\text { 一门艺术 善良过了头, 就成了软弱 } \\
\text { (Harshness or kindness is an art. } \\
\text { Extreme kindheartedness is } \\
\text { considered a kind of weakness) }\end{array}$ \\
\hline
\end{tabular}

TABLE III

THE SIZE OF DATA SETS FOR SENTIMENT ANALYSIS

\begin{tabular}{ccc}
\hline \hline source & $\begin{array}{c}\text { \# micro-blogs with } \\
\text { negative sentiment }\end{array}$ & $\begin{array}{c}\text { \# micro-blogs with } \\
\text { positive sentiment }\end{array}$ \\
\hline NLPCC & 8257 & 7134 \\
emoticons & 5783 & 14125 \\
\hline
\end{tabular}

1) The Distribution of Values of EI: We analyze the histogram of four dimensions of emotional intelligence in Figure 3 , and discover that they obey normal distribution.

The distribution area of the values of self-awareness is smaller to narrow than the values of self-motivation and mostly concentrate on the low-value region. The values of self-regulation are also distributed in the low-value region.

On the one hand, the deviation of identifying sentiment words results in lower values. On the other hand, users incline to publish more positive text. Therefore, the number of categories of sentiment words is less. The number of low values of social relationships is larger than high values, owing to the number of comments and retweet are small in most cases.

2) Gender Difference: Social role theory [48] is considered as the theoretical basis to explain the gender differences in behavior. Social role theory points out that gender differences in behavior is mainly due to the process of the social construction, and encode and process information in different patterns between men and women [49] [50]. Although gender differences have been found in previous work, in this study, we focus on the gender differences in EI.

We compare correlation coefficients across genders. We calculate the average values of four dimensions for different 
TABLE IV

THE RELATIONSHIP BETWEEN EI AND GENDER

\begin{tabular}{cccccc}
\hline \hline gender & $\mathrm{A}$ & $\mathrm{M}$ & $\mathrm{R}$ & $\mathrm{S}$ & sum \\
\hline female & 1.147648 & 0.605886 & 1.244395 & 0.479632 & 3.477561 \\
male & 1.040861 & 0.557913 & 1.215466 & 0.476139 & 3.290380 \\
\hline
\end{tabular}

A represents self-awareness, $M$ represents self-motivation, $R$ represents self-regulation, and $\mathrm{S}$ represents social relationships.

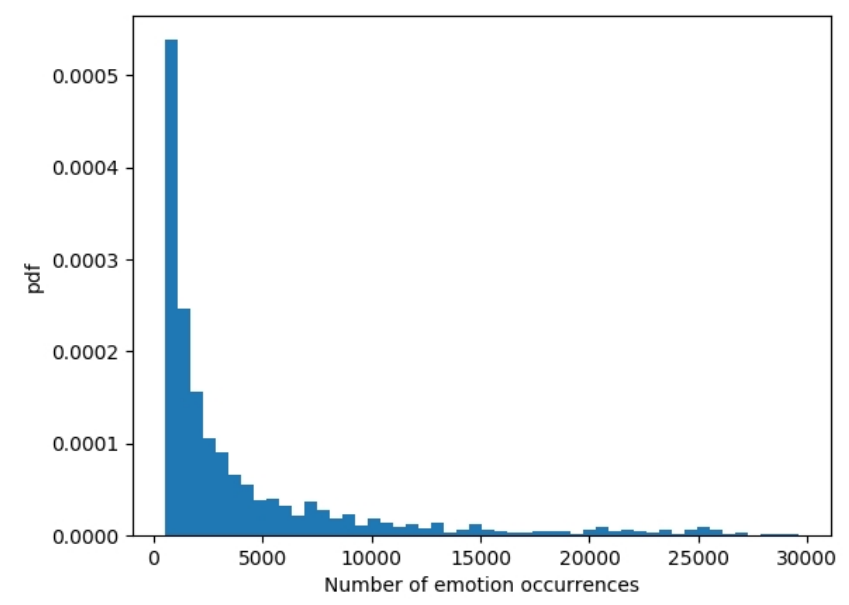

Fig. 2. Emoticons histogram. Pdf represents probability density function.

genders. From Table IV, we note a relationship between emotional intelligence and gender. The results show that EIs of females are higher than those of males. This supports the hypothesis that women have more emotional intelligence values than those of males. Intuitively, females are mostly expected to be more expressive of feelings in the culture. This result is in accordance with the previous work [51] [52] [53].

3) Correlation Coefficient Analysis: As shown in Figure 4 , the values of the four dimensions of emotional intelligence gradient descend. And obviously, the contours of the joint probability density of the self-awareness and the selfmotivation bend greatly. Its distribution is dense and decreases rapidly which implies a great difference in numerical value. However, the distribution of joint probability density of the self-regulation and the social relationships is sparse and decreases more slowly which implies a small change in numerical value. The positive correlation between the selfawareness and the self-motivation indicates that the person who has good cognition to emotions is always in a positive mood. The correlation between the self-motivation and the social relationships is not obvious.

Further, we analyze the Spearman's correlation and the Pearson's correlation. The Spearman's correlation assesses monotonic relationships, while the Pearson's correlation assesses linear relationships. From Table V, we can observe that the linear relationships of the four dimensions of EI other than the self-awareness and the self-motivation are not obvious. While monotonic relationships of the four dimensions of EI is significant. The results also accord with our hypothesis that the value of one dimension of EI tends to increase when the other increases.

\section{Discussion AND ANALYsis}

To the best of our knowledge, this is the first time that emotional intelligence prediction models are proposed based on social networking data. We mine potentially psychological information in the users' posts and interactive information. The models are based on sentiment analysis. Thus the stronger the motivation of users to express their emotions is, the better the predictive effect of the models will be. However, most users in social networks are motivated by social interaction and selfpresentation [54]. For instance, users post self-relevant information to interact with others, companies run advertisement campaigns, and journalists gather information and express ideas. Thus the models have limitations when users do not express their emotions. Meanwhile, the extracted features of the models depend on expert knowledge.

In addition, we use the naive Bayesian method to classify sentence-level sentiment. It cannot distinguish the subtle emotions in language expressions, such as sarcasm and ridicule. Therefore, identifying various emotions and emotional characteristics in a sentence is the focus of future research.

There exist work on the measurement of emotional intelligence based on the questionnaire [55] [56] [57] [58]. The measurement of EI remains controversial. To evaluate the validity of the prediction models of EI, we can use the cross-validation. We preliminarily analyze the relationship between EI and gender, the distribution of the values of EI and conduct the correlation analysis. In the future, we will compare the relationship between EI and personality. Additional, we plan to employ questionnaires to optimize the models and apply the models to twitter. Most of the current machine learning methods are supervised and require a lot of labeled data. Because of the limited labeled data, we didn't employ those methods. In looking for benchmark data, we plan to design questionnaires and get labeled data by crowd-sourcing. Meanwhile, we will employ some machine learning such as logistic regression to predict the emotional intelligence.

Predicting emotional intelligence can be applied to various scenarios such as assessment of professional competence, personnel training, prediction of personal behavior, and psychological research.

Furthermore, we implement the topic analysis and the cluster analysis to analyze the characteristics with different values of EI and enlighten the next work.

1) Topic Analysis: We rank sum of the four values to obtain the top 1000 high-score users and the last 1000 low-score users, then we use Latent Dirichlet Allocation(LDA) to retrieve 

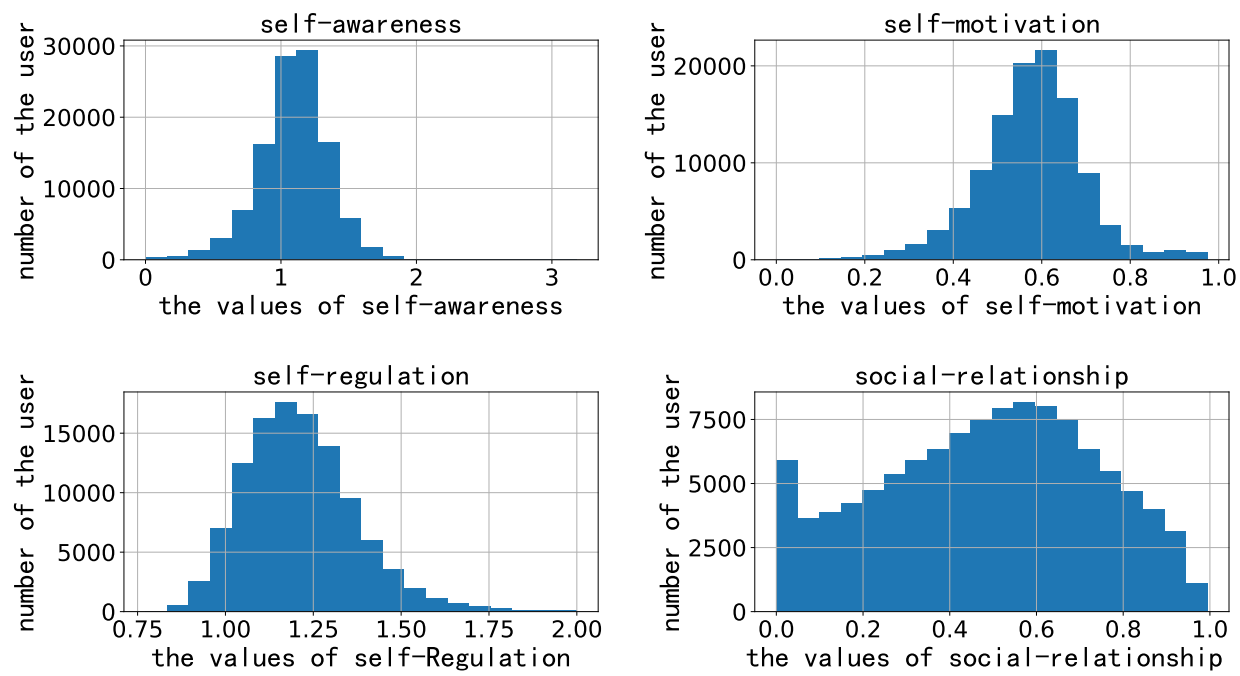

Fig. 3. The distribution of the four dimensions of emotional intelligence

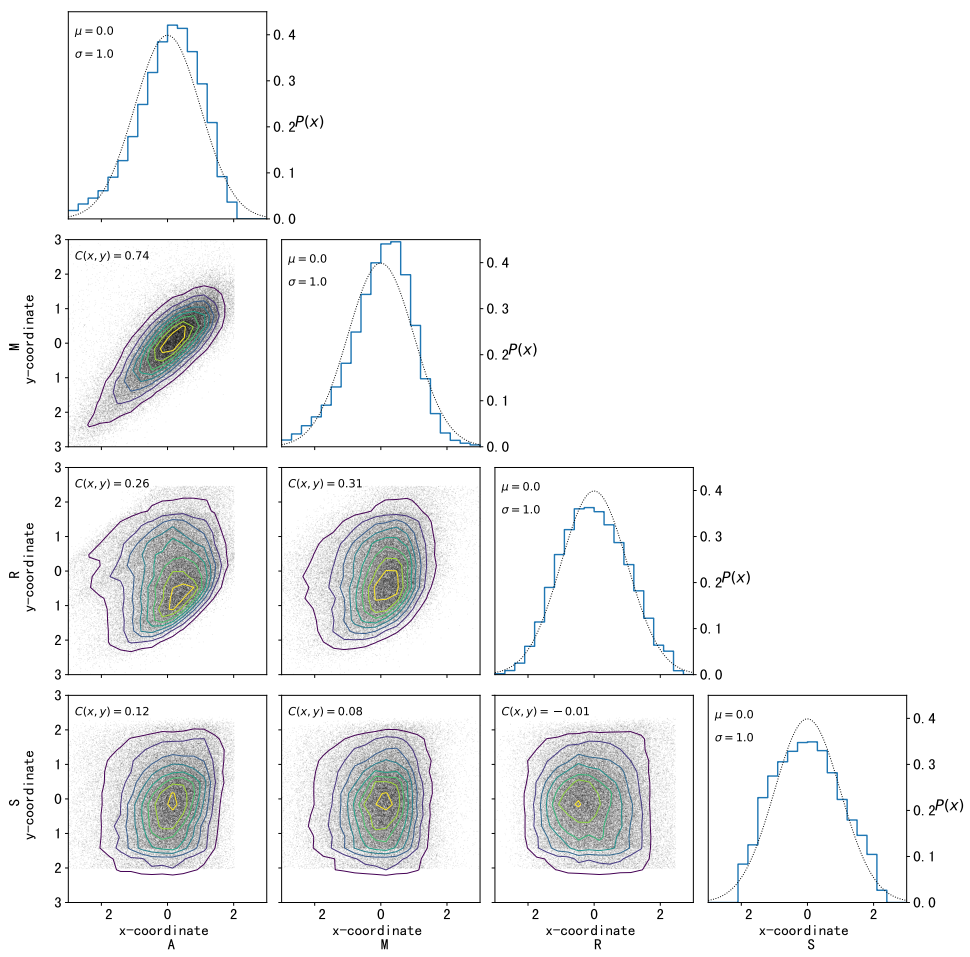

Fig. 4. Marginal distribution is used to analyze the relationship of the four dimensions of emotional intelligence. The diagonal panels show the marginal distributions, which corresponds to the respective mean and standard deviation, and the corresponding Gaussian distribution (dotted line). The off-diagonal panels show scatter-plots for the 2-dimensional distributions, contour-lines of equal density, and the correlation coefficients, $\mathrm{C}(\mathrm{x}, \mathrm{y})$.

key information in the two groups. Through contrasting the key top 30 words in 6 topics, we discover that the topics mostly concentrate on health, politics, economics and posts are much longer in low-score groups. However, the high-score users prefer to publish posts which reflect inner feeling compared with the low-score users. A part of words are shown in Table VI and Table VII. The results suggest that the stronger the motivation of users to express their emotions, the higher the users score. In other words, aiming at the user with different motivations, we can design different features based on the present models in the future.

2) Cluster Analysis: We use an unsupervised cluster algorithm, Gaussian Mixture Models (GMMs), to analyze the categories of emotional intelligence.

First, the Bayesian information criterion (BIC) is used to find the optimal number of the cluster. As shown in Figure $5 \mathrm{a}$, the number of cluster $N_{c}=18$ provides the optimal 
TABLE V

CORRELATION COEFFICIENT ANALYSIS

\begin{tabular}{c|cccc|cccc}
\hline \hline \multirow{2}{*}{ Correlation } & \multicolumn{4}{|c|}{ Pearson } & \multicolumn{4}{c}{ Spearman } \\
\cline { 2 - 9 } & $\mathrm{A}$ & $\mathrm{M}$ & $\mathrm{R}$ & $\mathrm{S}$ & $\mathrm{A}$ & $\mathrm{M}$ & $\mathrm{R}$ & $\mathrm{S}$ \\
\hline $\mathrm{A}$ & 1 & 0.7870 & 0.4468 & 0.1725 & 1 & 0.8762 & 0.8517 & 0.8743 \\
$\mathrm{M}$ & 0.7870 & 1 & 0.5157 & 0.0850 & 0.8762 & 1 & 0.8598 & 0.8732 \\
$\mathrm{R}$ & 0.4468 & 0.5157 & 1 & -0.0118 & 0.8517 & 0.8598 & 1 & 0.8800 \\
$\mathrm{~S}$ & 0.1725 & 0.0850 & -0.0118 & 1 & 0.8743 & 0.8732 & 0.8800 & 1 \\
\hline
\end{tabular}

A represents self-awareness, $M$ represents self-motivation, $R$ represents self-regulation, and $S$ represents social relationships.

TABLE VI

SIX TOPICS IN LOW-SCORE OF EI GROUP

\begin{tabular}{cc}
\hline \hline topic & words \\
\hline topic1 & we, nothing, ourselves, can, that is, life, people, health.... \\
topic2 & market, company, international, price, project, bank, US Dollar, gold... \\
topic3 & china, development, hospital, work, Japan, security, construction, reform... \\
topic4 & society, children, treatment, organization, investigation, impact, media, life... \\
topic5 & economy, China, investment, experts, food, real estate, sector, policy... \\
topic6 & USA, journalists, services, news, activities, support, reporting, about... \\
\hline
\end{tabular}

TABLE VII

SIX TOPICS IN HIGH-SCORE OF EI GROUP

\begin{tabular}{cc}
\hline \hline topic & words \\
\hline topic1 & photography, love, transfer, cake, Beijing, sun, good morning, tomorrow.... \\
topic2 & Hip-hop, watch, like, greedy, warm, fans, enjoy, opportunity... \\
topic3 & breeze, support, thank you, friends, flowers, efforts, expectations, sunshine... \\
topic4 & awesome, laugh, happiness, roses, wedding, wedding dress, music, cheers.... \\
topic5 & thank you, gifts, movies, studios, fun, evening, coffee, wishes... \\
topic6 & Aha, applause, constellation, Child Star, appreciation, simple, delicious, restaurant... \\
\hline
\end{tabular}

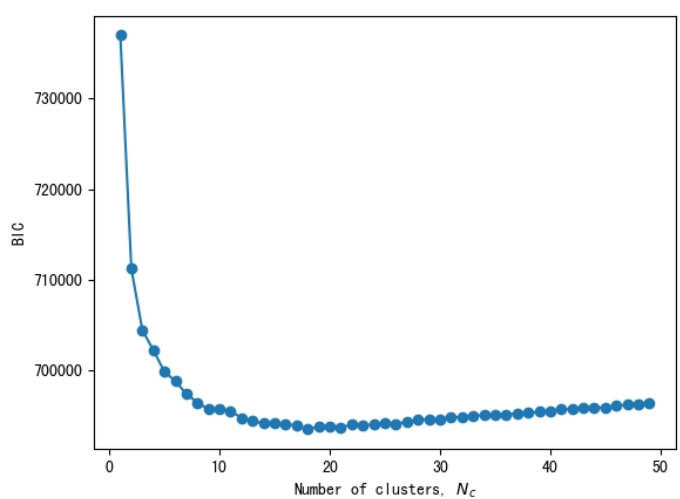

(a) The Bayesian information criterion (BIC) is used to find the optimal number of the cluster

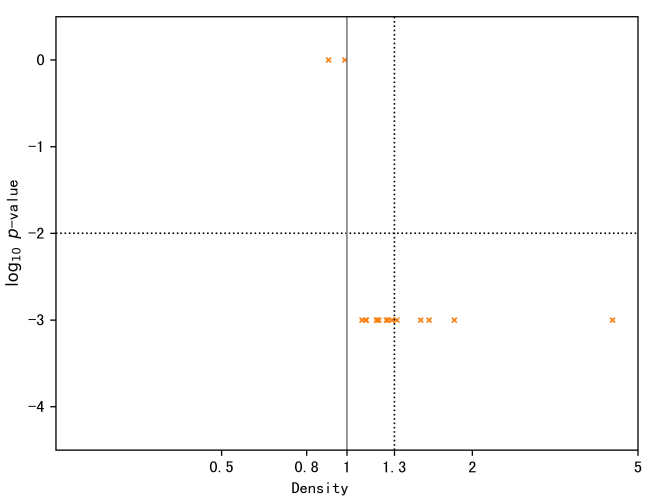

(b) Meaningful five clusters. We use Gaussian Mixture Models to find meaningful clusters
Fig. 5. Parameter selection for Gaussian Mixture Models

fit to the cluster. This number is much large, therefore we assess whether all identified clusters are truly meaningful. As is recommended [59], we estimate the density of each cluster. The p-value and the density are set to 0.01 and 1.3. Finally, as can be seen from Figure 5b, we identify five clusters. The five clusters are centered in the region in which we observe a larger fraction of person than expected from a randomized data set.

We analyze and compare characteristics of the five clusters in the Figure 6. Type A of emotional intelligence(ATEI) is characterized by the low value of self-regulation, compared with other dimensions of emotional intelligence with the high values. All dimensions of type $\mathrm{B}$ of emotional intelligence (BTEI) have high scores. Type $\mathrm{C}$ of emotional intelligence (CTEI) has high value of social relationships, however, the low score on self-awareness and self-motivation. In contrast to the characteristics of ATET, type D of emotional intelligence (DTEI) has low scores on all dimensions. Type E 

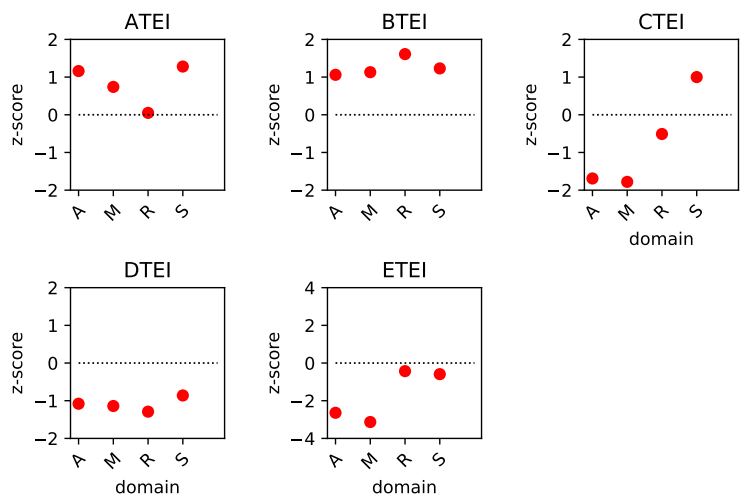

Fig. 6. The characteristics of the five clusters. ATEI, BTEI, CTEI, DTEI and ETEI are the five types of emotional intelligence.

of emotional intelligence (ETEI) has extremely low scores on self-awareness and self-motivation, however the scores of self-regulation and social relationships are close to average value. The characteristics of ATEI are similar to BTEI and are reverse to ETEI. Nevertheless, BTEI and DTEI show opposite characteristics.

We classify users from the perspective of emotional intelligence. The results indicate that users have different expressive habits. For instance, there is a lack of emotional expression in the posts of the user of CTEI. Thus, we can dynamically adjust weights of four dimensions of EI for summation according to different user's type to measure user's EI in the next work.

\section{CONClusion}

In this work, we propose prediction models to measure users' Emotional Intelligence (EI) through analyzing texts and interactive information in social networks. The models are expressed with four dimensions including self-awareness, selfregulation, self-motivation and social relationships. We test our models on one of largest social network of China, Weibo. In the experiments, we predict EIs of over a hundred thousand users in Weibo with four numerical values and the sum of these values. The experimental results show that the distribution of users' EIs is normal. The results also indicate that EIs of females are higher than those of males. In addition, we compare the topics between users with low scores and high scores and classify users from the perspective of emotional intelligence with the aim of further revealing the inherent law of users' emotional expression in social networks.

In future work, we plan to identify more emotions, design more fine-grained features to better predict users' emotional intelligence and conduct experiments on twitter data. Meanwhile, we will compare the relationship between EI and personality. Additional, we plan to employ questionnaires to optimize the models.

\section{REFERENCES}

[1] D. Jurgens, T. Finethy, J. McCorriston, Y. T. Xu, and D. Ruths, "Geolocation prediction in twitter using social networks: A critical analysis and review of current practice," in Ninth International AAAI Conference on Web and Social Media, 2015, pp. 188-197.
[2] U. Weinsberg, S. Bhagat, S. Ioannidis, and N. Taft, "Blurme: Inferring and obfuscating user gender based on ratings," in Proceedings of the sixth ACM conference on Recommender systems. ACM, 2012, pp. 195-202.

[3] T. Minkus, Y. Ding, R. Dey, and K. W. Ross, "The city privacy attack: Combining social media and public records for detailed profiles of adults and children," in Proceedings of the 2015 ACM on Conference on Online Social Networks. ACM, 2015, pp. 71-81.

[4] J. Burrus, A. Betancourt, S. Holtzman, J. Minsky, C. Maccann, and R. D. Roberts, "Emotional intelligence relates to well-being: Evidence from the situational judgment test of emotional management," Applied Psychology: Health and Well-Being, vol. 4, no. 2, pp. 151-166, 2012.

[5] J. Chen, Y. Liu, and M. Zou, "User emotion for modeling retweeting behaviors," Neural Networks, vol. 96, pp. 11-21, 2017.

[6] M. Kosinski, Y. Bachrach, P. Kohli, D. Stillwell, and T. Graepel, "Manifestations of user personality in website choice and behaviour on online social networks," Machine Learning, vol. 95, no. 3, pp. 357-380, 2014.

[7] M. Kosinski, D. Stillwell, and T. Graepel, "Private traits and attributes are predictable from digital records of human behavior," Proceedings of the National Academy of Sciences of the United States of America, vol. 110 , no. 15 , pp. 5802-5805, 2013.

[8] K. Modarresi, "Recommendation system based on complete personalization," Procedia Computer Science, vol. 80, pp. 2190-2204, 2016.

[9] T. Yarkoni and J. Westfall, "Choosing prediction over explanation in psychology: Lessons from machine learning," Perspectives on Psychological Science, vol. 12, no. 6, pp. 1100-1122, 2017.

[10] X. Liu, J. Liu, W. Wang, Y. He, and X. Zhang, "Discovering and understanding android sensor usage behaviors with data flow analysis," World Wide Web, vol. 21, no. 1, pp. 105-126, 2018.

[11] X. Liu, J. Liu, S. Zhu, W. Wang, and X. Zhang, "Privacy risk analysis and mitigation of analytics libraries in the android ecosystem," IEEE Transactions on Mobile Computing, pp. 1-17, 2019. [Online]. Available: https://doi.org/10.1109/TMC.2019.2903186

[12] W. Wang, M. Zhao, Z. Gao, G. Xu, H. Xian, Y. Li, and X. Zhang, "Constructing features for detecting android malicious applications: Issues, taxonomy and directions," IEEE Access, pp. 67 602-67631, 2019.

[13] W. Wang, J. Liu, G. Pitsilis, and X. Zhang, "Abstracting massive data for lightweight intrusion detection in computer networks," Information Sciences, vol. 433, pp. 417-430, 2018.

[14] W. Wang, X. Wang, D. Feng, J. Liu, Z. Han, and X. Zhang, "Exploring permission-induced risk in android applications for malicious application detection," IEEE Transactions on Information Forensics and Security, vol. 9, no. 11, pp. 1869-1882, 2014.

[15] W. Wang, Z. Gao, M. Zhao, Y. Li, J. Liu, and X. Zhang, "Droidensemble: Detecting android malicious applications with ensemble of string and structural static features," IEEE Access, vol. 6, pp. 31 798-31 807, 2018.

[16] W. Wang, T. Guyet, R. Quiniou, M.-O. Cordier, F. Masseglia, and X. Zhang, "Autonomic intrusion detection: Adaptively detecting anomalies over unlabeled audit data streams in computer networks," Knowledge-Based Systems, vol. 70, pp. 103-117, 2014.

[17] W. Wang, X. Guan, and X. Zhang, "Processing of massive audit data streams for real-time anomaly intrusion detection," Сотриter соттиnications, vol. 31, no. 1, pp. 58-72, 2008. 
[18] W. Wang, M. Zhao, and J. Wang, "Effective android malware detection with a hybrid model based on deep autoencoder and convolutional neural network," Journal of Ambient Intelligence and Humanized Computing, vol. 10, no. 8, pp. 3035-3043, 2019.

[19] W. Wang, Y. Shang, Y. He, Y. Li, and J. Liu, "Botmark: Automated botnet detection with hybrid analysis of flow-based and graph-based traffic behaviors," Information Sciences, 2019. [Online]. Available: https://doi.org/10.1016/j.ins.2019.09.024

[20] X. Wang, W. Wang, Y. He, J. Liu, Z. Han, and X. Zhang, "Characterizing android apps' behavior for effective detection of malapps at large scale," Future Generation Comp. Syst., vol. 75, pp. 30-45, 2017. [Online]. Available: https://doi.org/10.1016/j.future.2017.04.041

[21] N. Xie, X. Wang, W. Wang, and J. Liu, "Fingerprinting android malware families," Frontiers Comput. Sci., vol. 13, no. 3, pp. 637-646, 2019. [Online]. Available: https://doi.org/10.1007/s11704-017-6493-y

[22] L. Li, J. Liu, L. Cheng, S. Qiu, W. Wang, X. Zhang, and Z. Zhang, "Creditcoin: A privacy-preserving blockchain-based incentive announcement network for communications of smart vehicles," IEEE Trans. Intelligent Transportation Systems, vol. 19, no. 7, pp. 2204-2220, 2018. [Online]. Available: https://doi.org/10.1109/TITS.2017.2777990

[23] W. Wang, Y. Li, X. Wang, J. Liu, and X. Zhang, "Detecting android malicious apps and categorizing benign apps with ensemble of classifiers," Future Generation Computer Systems, vol. 78, pp. 987-994, 2018.

[24] G. Xu, Y. Zhang, A. K. Sangaiah, X. Li, A. Castiglione, and X. Zheng, "Csp-e2: An abuse-free contract signing protocol with low-storage ttp for energy-efficient electronic transaction ecosystems," Information Sciences, vol. 476, pp. 505-515, 2019.

[25] G. Xu, J. Liu, Y. Lu, X. Zeng, Y. Zhang, and X. Li, "A novel efficient maka protocol with desynchronization for anonymous roaming service in global mobility networks," Journal of Network and Computer Applications, vol. 107, pp. 83-92, 2018.

[26] X. Zeng, G. Xu, X. Zheng, Y. Xiang, and W. Zhou, "E-aua: An efficient anonymous user authentication protocol for mobile iot," IEEE Internet of Things Journal, vol. 6, no. 2, pp. 1506-1519, 2018.

[27] E. L. Thorndike, "Intelligence and its uses." Concours M 'dical, vol. 72, no. 18 , pp. $227-235,1920$.

[28] W. P. Alexander, "Intelligence, concrete and abstract: Note," British Journal of Psychology, vol. 29, no. 1, pp. 74-74, 1938.

[29] H. Gardner, "The theory of multiple intelligences," Ann Dyslexia, vol. 37, no. 1, pp. 19-35, 1987.

[30] P. Salovey and J. D. Mayer, "Emotional intelligence," Imagination Cognition Personality, vol. 9, no. 6, pp. 217-236, 1990.

[31] R. Bar-On, "The development of an operational concept of psychological well-being," Ph.D. dissertation, Rhodes University, 1985.

[32] J. D. Mayer, P. Salovey, and D. Caruso, "Models of emotional intelligence," RJ Sternberg (ed.), The handbook of intelligence, pp. 386-420, 2000.

[33] S. K. Davis and N. Humphrey, "Emotional intelligence predicts adolescent mental health beyond personality and cognitive ability," Personality Individual Differences, vol. 52, no. 2, pp. 144-149, 2012.

[34] M. Ferrando, M. D. Prieto, L. S. Almeida, C. Ferrándiz, R. Bermejo, J. A. López-Pina, D. Hernández, M. Sáinz, and M.-C. Fernández, "Trait emotional intelligence and academic performance: Controlling for the effects of iq, personality, and self-concept," Journal of Psychoeducational Assessment, vol. 29, no. 2, pp. 150-159, 2011.

[35] D. K. J. Gardner and P. Qualter, "Concurrent and incremental validity of three trait emotional intelligence measures," Australian Journal of Psychology, vol. 62, no. 1, pp. 5-13, 2011.

[36] D. L. Joseph and D. A. Newman, "Emotional intelligence: an integrative meta-analysis and cascading model," J Appl Psychol, vol. 95, no. 1, pp. 54-78, 2010.

[37] E. H. O. Jr, R. H. Humphrey, and J. M. Pollack, "The relation between emotional intelligence and job performance: A meta-analysis," Journal of Organizational Behavior, vol. 32, no. 5, pp. 788-818, 2011.

[38] B. R, "Baron emotional quotient inventory: Technical manaual," Toronto, ON: Multi-Health Systems Ins, 1997.

[39] X. Wei, J. Li, Z. Han, and W. Wang, "Predicting users' emotional intelligence with social networking data," in Proceedings of the 5th International Symposium on Security and Privacy in Social Networks and Big Data, 2019.

[40] D. Hillard, M. Ostendorf, and E. Shriberg, "Detection of agreement vs. disagreement in meetings: Training with unlabeled data," in Proceedings of the 2003 Conference of the North American Chapter of the Association for Computational Linguistics on Human Language Technology: companion volume of the Proceedings of HLT-NAACL 2003-short papers-Volume 2. Association for Computational Linguistics, 2003, pp. 34-36.
[41] C. Liebrecht, F. Kunneman, and A. van Den Bosch, "The perfect solution for detecting sarcasm in tweets\# not," in Proceedings of the 4th Workshop on Computational Approaches to Subjectivity, Sentiment and Social Media Analysis. New Brunswick, NJ: ACL, Atlanta, Georgia, USA, 2013, pp. 29-37.

[42] R. González-Ibánez, S. Muresan, and N. Wacholder, "Identifying sarcasm in twitter: a closer look," in Proceedings of the 49th Annual Meeting of the Association for Computational Linguistics: Human Language Technologies: Short Papers-Volume 2. Association for Computational Linguistics, 2011, pp. 581-586.

[43] T. H. Nguyen and K. Shirai, "Topic modeling based sentiment analysis on social media for stock market prediction," in Proceedings of the 53rd Annual Meeting of the Association for Computational Linguistics and the 7th International Joint Conference on Natural Language Processing (Volume 1: Long Papers), vol. 1, 2015, pp. 1354-1364.

[44] L. Xu, L. Hongfei, Y. Pan, H. Ren, and C. Jianmei, "Constructing the affective lexicon ontology," Journal of The China Society For Scientific and Technical Information, vol. 27, no. 2, pp. 180-185, 2008.

[45] X. Zhu, R. Ma, L. Sun, and H. Chen, "Word semantic similarity computeration based on hownet and cilin," Journal of Chinese Information Processing, vol. 30, no. 4, pp. 29-36, 2016.

[46] J. Bi and C. Zhang, "An empirical comparison on state-of-the-art multiclass imbalance learning algorithms and a new diversified ensemble learning scheme," Knowledge-Based Systems, vol. 158, pp. 81-93, 2018.

[47] C. Zhang, C. Liu, X. Zhang, and G. Almpanidis, "An up-to-date comparison of state-of-the-art classification algorithms," Expert Systems with Applications, vol. 82, pp. 128-150, 2017.

[48] A. H. Eagly, Sex differences in social behavior: A social-role interpretation. Psychology Press, 2013.

[49] S. L. Bem, "The bsri and gender schema theory: A reply to spence and helmreich.” Psychological Review, no. 4, pp. 369-371, 1981.

[50] V. Venkatesh and M. G. Morris, "Why don't men ever stop to ask for directions? gender, social influence, and their role in technology acceptance and usage behavior," MIS quarterly, pp. 115-139, 2000.

[51] E. Lopez-Zafra and L. Gartzia, "Perceptions of gender differences in self-report measures of emotional intelligence," Sex Roles, vol. 70, no. 11-12, pp. 479-495, 2014.

[52] N. S. Schutte, J. M. Malouff, L. E. Hall, D. J. Haggerty, J. T. Cooper, C. J. Golden, and L. Dornheim, "Development and validation of a measure of emotional intelligence," Personality Individual Differences, vol. 25 , no. 2 , pp. $167-177,1998$.

[53] D. L. D. V. S. Maria, M. Africa Borges del Rosal, N. Ruvalcaba Romero, K. Villegas, and M. Lorenzo, "Emotional intelligence and its relationship with gender, academic performance and intellectual abilities of undergraduates." Electronic Journal of Research in Educational Psychology, vol. 11, pp. 395-412, 2013.

[54] D. Zhang, X. Feng, and P. Chen, "Examining microbloggers' individual differences in motivation for social media use," Social Behavior and Personality, vol. 46, no. 4, pp. 667-682, 2018.

[55] J. D. Mayer, P. Salovey, D. R. Caruso, and G. Sitarenios, "Measuring emotional intelligence with the msceit v2.0." Emotion, vol. 3, no. 1, pp. 97-105, 2003.

[56] L. M. Wood, J. D. Parker, and K. V. Keefer, "Assessing emotional intelligence using the emotional quotient inventory (eq-i) and related instruments," in Assessing emotional intelligence. Springer, 2009, pp. 67-84.

[57] K. V. Petrides, "Psychometric properties of the trait emotional intelligence questionnaire (teique)," in Assessing emotional intelligence. Springer, 2009, pp. 85-101

[58] C. S. Wong, K. S. Law, and P. M. Wong, "Development and validation of a forced choice emotional intelligence measure for chinese respondents in hong kong," Asia Pacific Journal of Management, vol. 21, no. 4, pp. $535-559,2004$.

[59] M. Gerlach, B. Farb, W. Revelle, and L. A. N. Amaral, "A robust datadriven approach identifies four personality types across four large data sets," Nature human behaviour, vol. 2, no. 10, p. 735, 2018. 


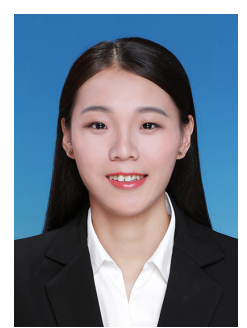

Xiangyu Wei received the B.S. degree from Shanxi University, China, in 2016. She is currently pursuing the Ph.D. degree with the School of Computer and Information Technology in Beijing Jiaotong University. Her main research interests include social networks analysis and information security.

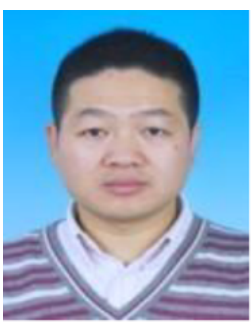

Guangquan Xu is a Ph.D. and full professor at the Tianjin Key Laboratory of Advanced Networking (TANK), College of Intelligence and Computing, Tianjin University, China. He received his Ph.D. degree from Tianjin University in March 2008. He is a member of the CCF and IEEE. His research interests include cyber security and trust management.

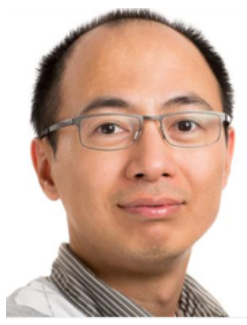

Hao Wang received the B.Eng. and Ph.D. degrees in computer science and engineering from the South China University of Technology, Guangzhou, China, in 2006. He is currently an Associate Professor with the Norwegian University of Science and Technology, Trondheim, Norway. He has authored or co-authored $80+$ papers in reputable international journals and conferences. His current research interests include big data analytics, industrial internet of things, high performance computing, safety-critical systems, and communication security.

Dr. Wang is a member of the IEEE IES Technical Committee on Industrial Informatics. He served as a TPC Co-Chair for the IEEE DataCom 2015, IEEE CIT 2017, and ES 2017. He served as a reviewer for journals such as the IEEE TRANSACTIONS ON KNOWLEDGE AND DATA ENGINEERING, the IEEE TRANSACTIONS ON INDUSTRIAL INFORMATICS, the IEEE TRANSACTIONS ON BIG DATA, the IEEE TRANSACTIONS ON EMERGING TOPICS IN COMPUTING, the IEEE TRANSACTIONS ON INFORMATION FORENSICS AND SECURITY, the IEEE INTERNET OF THINGS JOURNAL, and ACM Transactions on Multimedia Computing, Communications, and Applications.

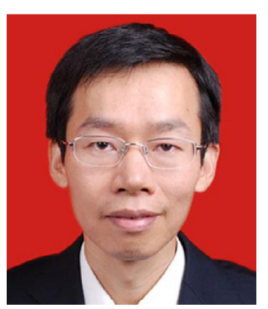

Yongzhong $\mathrm{He}$ is currently an Associate Professor with the School of Computer and Information Technology, Beijing Jiao tong University, Beijing, China. He earned his Ph.D.degree from Graduate School of Chinese Academy of Sciences in 2006 . He has authored or coauthored over 20 peer-reviewed papers in journals and conferences. His main research interests include system security and privacy.

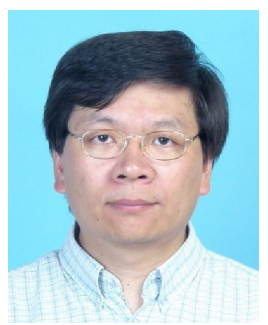

Zhen Han received his Ph.D. degree from China Academy of Engineering Physics, in 1991. He is currently a Professor at the School of Computer and Information Technology of Beijing Jiaotong University. He has authored or co-authored over 100 papers in various journals and international conference. His main research interests are information security architecture and trusted computing.

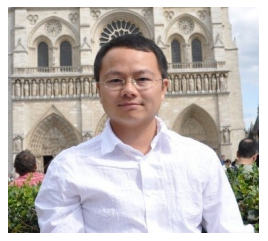

Wei Wang is currently full professor in the School of Computer and Information Technology, Beijing Jiaotong University, China. He earned his Ph.D. degree in control science and engineering from $\mathrm{Xi}^{\text {" }}$ an Jiaotong University, China, in 2006. He was a postdoctoral researcher in University of Trento, Italy, from 2005 to 2006 . He was a postdoctoral researcher in TELECOM Bretagne and in INRIA, France, from 2007 to 2008. He was a European ERCIM Fellow in Norwegian University of Science and Technology (NTNU), Norway, and in Interdisciplinary Centre for Security, Reliability and Trust (SnT), University of Luxembourg, from 2009 to 2011. He visited INRIA, ETH, NTNU, CNR, and New York University Polytechnic. He is an editorial board member for Computer \& Security and a young AE of Frontiers of Computer Science Journal. He has authored or co-authored over 90 peer-reviewed papers in various journals and international conferences. His main research interests include mobile, computer and network security. 